\title{
Optimum Modulator Design for a High-Sensitivity Homodyne System-Binary Modulation Method
}

\author{
KENZO WATANABE, MEMBER, IEEE, AND IWAO TAKAO, MEMBER, IEEE
}

\begin{abstract}
The optimum modulation method for a high-sensitivity homodyne system employing a reflection-type modulator is investigated. General expressions are derived which describe the effect of the modulator performance on the phase-sensitive conversion loss and noise figure of the system. It is shown that the highest phasedetection and the highest amplitude-detection sensitivities can be simultaneously attainable by the binary modulation method. This theoretical result is confirmed by the performance of the practical system employing a varactor binary modulator. The system has the minimum conversion loss of $6.3 \mathrm{~dB}$. The rejection ratio for the quadrature signal is $42 \mathrm{~dB}$, and the noise figure is $8.7 \mathrm{~dB}$ at $9.45 \mathrm{GHz}$.
\end{abstract}

\section{INTRODUCTION}

$\mathbf{T}$ HE homodyne detection system employing a signal modulation technique [1], or homodyne system for short, has been widely used for the phase-sensitive detection of microwave signals in laboratory experiments [2][8] and in industrial systems [9], [10]. The system originated from a direct-detection phase-sensitive detector, but unlike the video detection used in direct-detection systems, an IF detection is employed in homodyne systems, made possible because a modulated signal can be mixed with the original carrier. The essential feature of the homodyne system is the high sensitivities for both phase and amplitude detections. Many authors have analyzed the modulation-detection process of the homodyne system [1], [6]-[8]. However, the process treated in their analyses was only the amplitude modulation and the envelope detection, and no investigation of the modulation-detection method to increase the detection sensitivity has been made.

The purpose of the work described in this paper is to investigate what is the best modulation method for a high-sensitivity homodyne system. A general expression for the conversion loss is first derived which describes the phase-sensitive detection of the homodyne system. Then, using this expression and the phasor diagram of the instantaneous first sideband of the modulated signal, possible modulation methods which provide the highest rejection ratio for the quadrature signal are investigated. This rejection ratio is used as the measure of the phase-detection sensitivity of the homodyne system throughout the paper. Next, the modulation method which provides the homodyne system with the highest amplitude-detection sensitivity is examined by deriving the expression for the noise

Manuscript received November 20, 1973; revised May 20, 1974. $\mathrm{K}$. Watanabe is with the Research Institute of Electronics, Shizuoka University, Hamamatsu 432, Japan.

I. Takao was with the Research Institute of Electronics, Shizuok University, Hamamatsu 432, Japan. He is now with the North Shore College, Atsugi 243, Japan. figure of the system. These theoretical studies show that the binary modulation method is optimum for the highsensitivity homodyne system. Finally, experimental verifications are described.

\section{THEORETICAL ANALYSIS OF A HOMODYNE SYSTEM}

\section{A. Derivation of Conversion Loss}

A basic circuit configuration of a homodyne system is shown in Fig. 1. Power from a microwave source at angular frequency $\omega$ is divided between the carrier and the signal channels. The wave in the carrier channel, the homodyne carrier, is led to the mixer after being attenuated to an appropriate level and excites the mixer diodes. The wave in the signal channel is led to the unknown circuit or material whose property is to be measured, and is subjected to amplitude or phase variations. This signal is modulated by a reflection-type modulator driven at the angular frequency $\omega_{m}$, much lower than $\omega$, and then mixed with the homodyne carrier to be converted to the IF signal at angular frequency $\omega_{m}$. After being amplified, the IF signal is detected by a low-frequency detector which generates the video signal.

The complex reflection coefficient $\rho$ of the modulator can be described by its real and imaginary parts. Expanding each part into a Fourier series at fundamental angular frequency $\omega_{m}$, one has

$$
\begin{aligned}
\rho=\operatorname{Re}\{\rho\}+j \operatorname{Im}\{\rho\}=\sum_{n=0}^{\infty} & \left\{a_{n} \cos \left(n \omega_{m} t+\theta_{n}\right)\right. \\
& \left.+j b_{n} \cos \left(n \omega_{m} t+\phi_{n}\right)\right\} .
\end{aligned}
$$

The microwave signal from the unknown is

$$
e_{s}=\operatorname{Re}\left\{V_{s} \exp [j(\omega t+\psi)]\right\} .
$$

The modulated signal $e_{m}$ is given by

$$
\begin{aligned}
e_{m}= & \operatorname{Re}\left[\rho e_{s}\right] \\
= & \left(V_{s} / 2\right) \sum_{n=0}^{\infty}\left\{\left[a_{n}^{2}+b_{n}^{2}+2 a_{n} b_{n} \sin \left(\theta_{n}-\phi_{n}\right)\right]^{1 / 2}\right. \\
& \cdot \cos \left[\left(\omega+n \omega_{m}\right) t+\psi+\alpha_{n}\right] \\
& +\left[a_{n}^{2}+b_{n}^{2}-2 a_{n} b_{n} \sin \left(\theta_{n}-\phi_{n}\right)\right]^{1 / 2} \\
& \left.\cdot \cos \left[\left(\omega-n \omega_{m}\right) t+\psi-\beta_{n}\right]\right\}
\end{aligned}
$$

1 The representations of the reflection coefficient by (1) and the modulated signal by (3) assume a linear time-varying modulator However, they hold for a nonlinear modulator as long as the sign level and the modulation frequency stay, respectively, much lo than the modulator driving level and the signal frequency. 


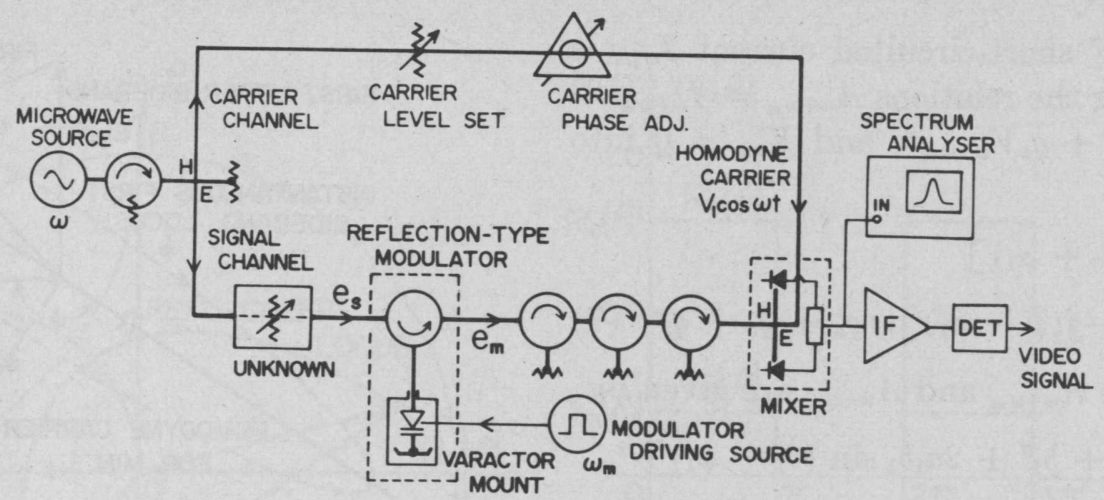

Fig. 1. The homodyne system.

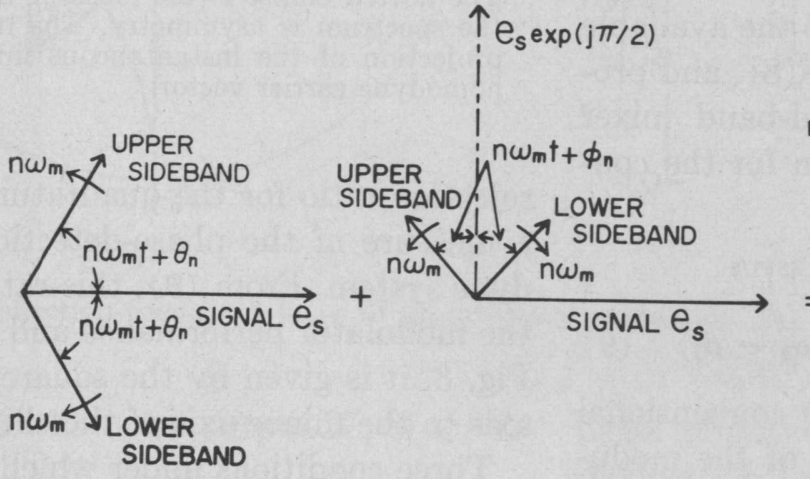

(a)

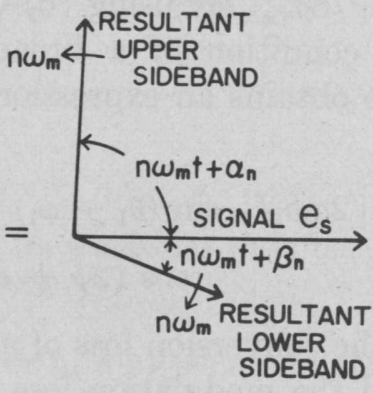

(c)

Fig. 2. The phasor diagram of $n$th upper and lower sidebands generated by variations of (a) the real part, and of (b) the imaginary part of the reflection coefficient, and (c) the resultant upper and lower sidebands.

where

$$
\begin{aligned}
& \alpha_{n}=\tan ^{-1} \frac{a_{n} \sin \theta_{n} \pm b_{n} \cos \phi_{n}}{a_{n} \cos \theta_{n} \mp b_{n} \sin \phi_{n}} \\
& \beta_{n}=\theta_{n} \pm \tan ^{-1} \frac{b_{n} \cos \left(\theta_{n}-\phi_{n}\right)}{a_{n} \pm b_{n} \sin \left(\theta_{n}-\phi_{n}\right)}
\end{aligned}
$$

Elaborating on the modulation process, the phasor diagram of $n$th upper and lower sidebands is shown in Fig. 2. The signal is represented as a fixed-reference vector by reducing the whole angular velocity of vectors by the signal angular frequency $\omega$. On this stationary phasor diagram, the $n$th sideband vectors rotate with angular $\phi_{n}$; the resultant upper and lower sidebands differ in magnitude as shown in Fig. 2(c), which checks with (3). Thus, when both real and imaginary parts of the reflection coefficient vary periodically, the signal is simultaneously amplitude- and angle-modulated, and the spectrum becomes asymmetrical with respect to the signal.

In deriving the relation between the terminal voltage and the current of the mixer, the following two assumptions are made: the mixer diode is a nonlinear conductance device which is excited by the homodyne carrier $V_{1} \cos \omega t$. It is only the first upper and lower sidebands that produce the IF component of $\omega_{m}$ by being mixed with the homodyne carrier. On applying the standard mixer theory [11], one can obtain the following relation

$$
\left[\begin{array}{c}
I_{\omega+\omega_{m}} \\
I_{\omega_{m}} \\
I_{\omega-\omega_{m}} *
\end{array}\right]=\left[\begin{array}{ccc}
g_{0} & g_{1} \exp \left[-j\left(\psi+\alpha_{1}\right)\right] & g_{2} \exp \left[-j\left(2 \psi+\alpha_{1}-\beta_{1}\right)\right] \\
g_{1} \exp \left[j\left(\psi+\alpha_{1}\right)\right] & g_{0} & g_{1} \exp \left[-j\left(\psi-\beta_{1}\right)\right] \\
g_{2} \exp \left[j\left(2 \psi+\alpha_{1}-\beta_{1}\right)\right] & g_{1} \exp \left[j\left(\psi-\beta_{1}\right)\right] & g_{0}
\end{array}\right]\left[\begin{array}{c}
V_{\omega+\omega_{m}} \\
V_{\omega_{m}} \\
V_{\omega-\omega_{m}}{ }
\end{array}\right]
$$

velocities $\pm n \omega_{m}$. The real part of the reflection coefficient produces the sideband pair shown in Fig. 2(a), the phasor sum of which yields a resultant vector collinear with the signal vector, just like the sidebands in an amplitude modulation. The imaginary part produces the sideband pair shown in Fig. 2(b), which yields a resultant vector in quadrature with the signal vector, just like the oddorder sidebands in an angle modulation. The phase angle between upper sidebands in Fig. 2 (a) and (b) is $\frac{1}{2} \pi-$ $\theta_{n}+\phi_{n}$, while that between lower sidebands is $\frac{1}{2} \pi+\theta_{n}-$ where $g_{n}$ when $(n=0,1,2$,$) are the n$th Fourier coefficients of the differential conductance of the mixer diode, and the asterisk denotes the complex conjugate.

The IF output conductance $g_{\omega_{m}}$ can be found by introducing the relations $I_{\omega+\omega_{m}}=-g_{a} V_{\omega+\omega_{m}}, I_{\omega-\omega_{m}} *$ $-g_{a} V_{\omega-\omega_{m}}{ }^{*}$, and $I_{\omega_{m}}=g_{\omega_{m}} V_{\omega_{m}}$ into (5). Solving for $g_{\omega_{m}}$ gives

$$
g_{\omega_{m}}=g_{0}-2 g_{1}^{2} / g_{a}+g_{0}+g_{2}
$$

where $g_{a}$ is the characteristic conductance of the signal 
transmission line. The IF short-circuited current $I_{\omega_{m} s}$ is next found by introducing the relations $A_{\omega+\omega_{m}}=I_{\omega+\omega_{m}}+$ $g_{a} V_{\omega+\omega_{m}}, A_{\omega-\omega_{m}}=I_{\omega-\omega_{m}}{ }^{*}+g_{a} V_{\omega-\omega_{m}}{ }^{*}$, and $V_{\omega_{m}}=0$ into (5). Solving for $I_{\omega_{m}}$ gives

$$
\begin{aligned}
I_{\omega_{m} s}=g_{1}\left\{A_{\omega+\omega_{m}} \exp \left[j\left(\psi+\alpha_{1}\right)\right]\right. \\
\left.\quad+A_{\omega-\omega_{m}} \exp \left[-j\left(\psi-\beta_{1}\right)\right]\right\} / g_{a}+g_{0}+q_{2}
\end{aligned}
$$

where the current sources $A_{\omega+\omega_{m}}$ and $A_{\omega-\omega_{m}}$ are given by

$$
A_{\omega \pm \omega_{m}}=\left(g_{a} V_{s} / 2\right)\left[a_{1}^{2}+b_{1}^{2} \pm 2 a_{1} b_{1} \sin \left(\theta_{1}-\phi_{1}\right)\right]^{1 / 2} \text {. }
$$

The conversion loss of a homodyne system is defined as the ratio of the signal power $S_{i}=g_{a} V_{s}^{2} / 8$ to the available IF power $S_{i f}=\left|I_{\omega_{m} s}\right|^{2} / 8 g_{\omega_{m}}$. By using (6)-(8), and providing the matching condition of a broad-band mixer $g_{a}=g_{0}-g_{2}[11]$, one obtains an expression for the conversion loss

$$
\begin{aligned}
& L_{h}=L_{c} L_{m} / 1+\left\{1-\left[2 a_{1} b_{1} L_{m}\right.\right.\left.\left.\sin \left(\theta_{1}-\phi_{1}\right)\right]^{2}\right\}^{1 / 2} \\
& \cdot \cos \left(2 \psi+\alpha_{1}-\beta_{1}\right)
\end{aligned}
$$

where $L_{c}$ and $L_{m}$ are the conversion loss of a conventional broad-band mixer and the modulation loss of the modulator, respectively, and are given by

$$
\begin{aligned}
& L_{c}=4 g_{0}\left(g_{0}{ }^{2}-g_{1}{ }^{2}\right) / g_{1}{ }^{2}\left(g_{0}-g_{2}\right) \\
& L_{m}=2 / a_{1}{ }^{2}+b_{1}{ }^{2} .
\end{aligned}
$$

\section{B. Phase-Detection Sensitivity and Modulation Method}

The conversion loss $L_{h}$ becomes minimum when $2 \psi+$ $\alpha_{1}-\beta_{1}=2 \nu \pi$, and maximum when $2 \psi+\alpha_{1}-\beta_{1}=$ $(2 \nu+1) \pi$, where $\psi$ is the phase difference between the signal and the homodyne carrier, and $\nu$ is an integer. This phase-sensitive conversion loss is phenomenologically explained by the phasor diagram shown in Fig. 3. The reference axis is also the stationary signal vector. The homodyne carrier is then a stationary vector which makes an angle $\psi$ with respect to the reference axis. The sideband vectors shown in Fig. 2(c) are reproduced as first upper and lower sidebands, each rotating with angular frequencies $\pm \omega_{m}$ relative to the signal. The phasor sum of these two sidebands yields the instantaneous sideband vector, which rotates with the angular frequency $\omega_{m}$ with the time-varying amplitude and, due to the asymmetrical spectrum, traces out an elliptic orbit, as shown by the dotted curve. Since the instantaneous IF signal is the projection of the instantaneous sideband vector on the homodyne carrier axis, the IF voltage becomes maximum when the homodyne carrier is collinear with the major axis of the ellipse, and minimum when it is collinear with the minor axis. Considering this situation from the viewpoint of the homodyne carrier being fixed instead of the signal, the conversion loss varies with the signal phase.

Suppose now the microwave signal consists of two components with the same amplitude but in phase quadrature. When the conversion loss for one component is minimum, that for the other is maximum. The ratio of the maximum to the minimum in the conversion loss is defined as the

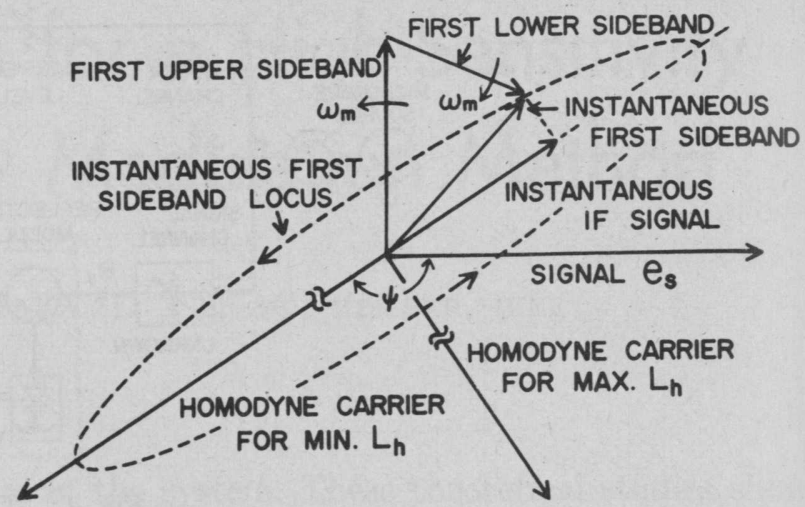

Fig. 3. The phasor diagram of the instantaneous first sideband The dotted ellipse is the locus of the first sideband vector when the spectrum is asymmetry. The instantaneous IF signal is the projection of the instantaneous first sideband vector along the homodyne carrier vector.

rejection ratio for the quadrature signal, which is used as a measure of the phase-detection sensitivity of a homodyne system. From (9), this ratio is solely determined by the modulator performance and, in the phasor diagram of Fig. 3, it is given by the square of the ratio of the major axis to the minor axis of the ellipse.

Three conditions under which the quadrature signal rejection ratio becomes infinity are present: 1) $a_{1}=0$, 2) $b_{1}=0$, and 3) $\theta_{1}-\phi_{1}=\nu \pi$. Then (9) is simplified as

$$
L_{h}=L_{c} L_{m} / 2 \cos ^{2}\left[\psi+\left(\alpha_{1}-\beta_{1}\right) / 2\right] .
$$

Condition 1) is met by phase modulation without any amplitude modulation, and condition 2) is met by amplitude modulation without any phase modulation. The loci of the instantaneous first sideband are then straight lines 1) perpendicular to, and 2) collinear with, the signal vector, respectively. However, in practice, the realization of these two modulation methods in the microwave frequency region is difficult since the phase modulation is inevitably accompanied, more or less, by the amplitude modulation, and, conversely, the amplitude modulation is inevitably accompanied by the phase modulation.

Condition 3) means that the real and the imaginary parts of the reflection coefficient must be varied just in phase or just in antiphase when both of them coexist. The locus of the instantaneous first sideband then becomes a straight line making an angle $\tan ^{-1}\left(b_{1} / a_{1}\right)$ with respect to the signal vector, as seen from (3) and (4). Practically, this condition is met by the binary modulation method, which is explained in Fig. 4: when a modulator with an arbitrary reflection-coefficient locus as shown in Fig. 4(a) is driven so that it takes $\rho_{a}$ and $\rho_{b}$ in every half-period of the modulation frequency, the real and imaginary parts vary just in phase as shown in Fig. 4 (b). The binary modulation method is superior to phase and amplitude modulation methods in that it is applicable to any modulator, and this simplifies the design of a modulator.

\section{Noise Figure and Modulation Loss}

The conversion loss for the microwave incoherent noise incident on the homodyne system is $L_{c} L_{m}$, as seen from $(9)$, while that for the noise originated in the mixer diode 


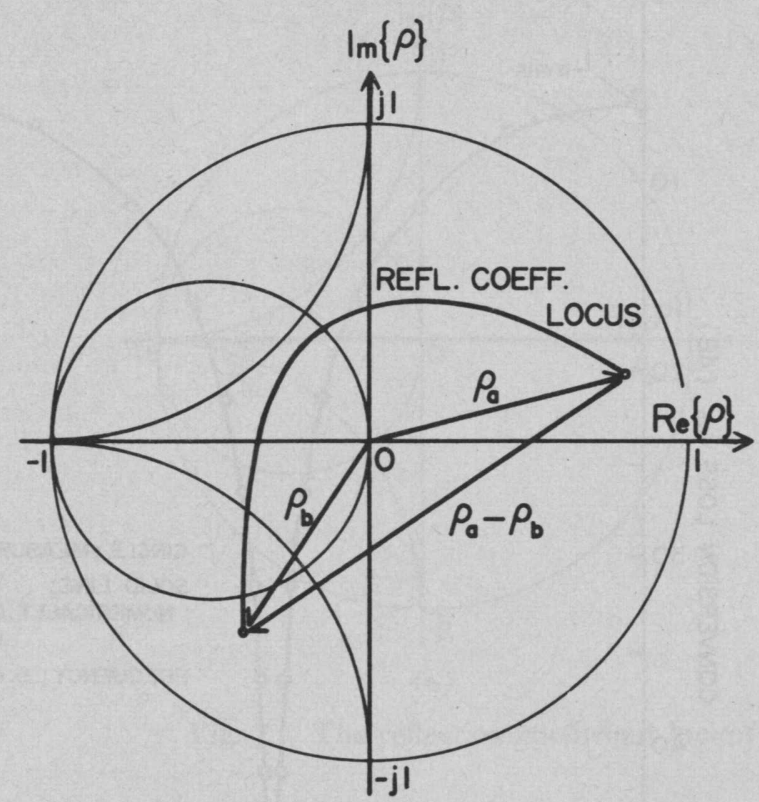

(a)

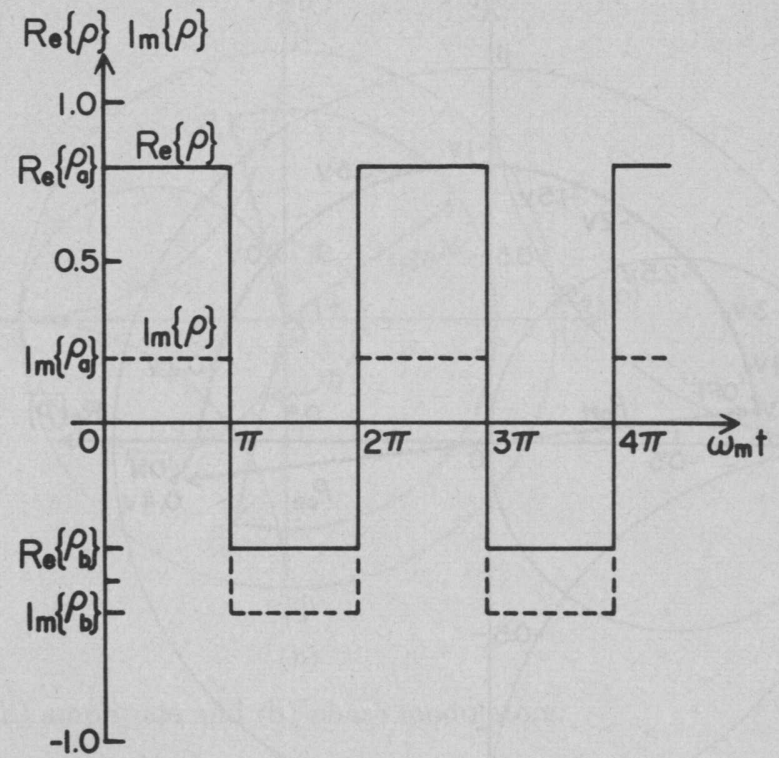

(b)

Fig. 4. (a) The reflection-coefficient locus of an arbitrary modulator. (b) Its waveforms when binary-modulated.

is $L_{c}$. With these in mind, the noise output $N_{o}$ of an IF amplifier is given by

$$
N_{o}=\left[N_{i} / L_{m}+\left(F_{\text {rec }}-1\right) k T_{0} B\right]\left(G_{\mathrm{if}} / L_{c}\right)
$$

where $N_{i}$ is the incident microwave noise power, $F_{\text {rec }}$ is the noise figure of the receiver consisting of the mixer and the IF amplifier, and $B$ and $G_{\mathrm{if}}$ are the bandwidth and the gain of the IF amplifier, respectively. On using (12), the signal output power $S_{o}$ is given by

$$
S_{o}=2 G_{\mathrm{if}} S_{i} \cos ^{2}\left[\psi+\left(\alpha_{1}-\beta_{1}\right) / 2\right] / L_{c} L_{m} .
$$

Thus the output signal-to-noise ratio is

$$
\frac{S_{o}}{N_{o}}=\frac{2 S_{i} \cos ^{2}\left[\psi+\left(\alpha_{1}-\beta_{1}\right) / 2\right]}{N_{i}+L_{m}\left(F_{\text {rec }}-1\right) k T_{0} B} .
$$

If the output noise is contributed mainly from the incident microwave noise, (15) becomes the same as that for the double-sideband detection [12], and a maximum improvement of $3 \mathrm{~dB}$ in the signal-to-noise ratio is possible.

The frequency-transformation process of the modulator doubles the bandwidth for the incident thermal noise. Putting $N_{i}=2 k T_{0} B$, and taking the ratio of input-output signal-to-noise ratio, one finds a noise figure expression of the homodyne system

$$
F=\frac{1+L_{m}\left(F_{\mathrm{rec}}-1\right) / 2}{\cos ^{2}\left[\psi+\left(\alpha_{1}-\beta_{1}\right) / 2\right]}
$$

It is noted that lowering the modulation loss is of prime importance in designing a low-noise homodyne system.

Referring to Fig. 4, the modulation loss of the binary modulator is given by

$$
L_{m}=\left(\pi / \sqrt{2}\left|\rho_{a}-\rho_{b}\right|\right)^{2} .
$$

Equation (17) takes the minimum value $\pi^{2} / 8$ or $0.91 \mathrm{~dB}$ when the ideal binary $180^{\circ}$ modulation $\left|\rho_{a}-\rho_{b}\right|=2$ is realized. Since any practical modulator, whether it may be an amplitude or a phase modulator, provides the lowest modulation loss when binary modulated, as described in the Appendix, the binary modulation method is superior also in obtaining a high amplitude-detection sensitivity.

\section{EXPERIMENTAL RESULT}

The homodyne system has been constructed for the experimental verification of the theory described in the previous section. The arrangement of the system is the same as that shown in Fig. 1, except that the variable attenuator is inserted in the signal channel instead of the unknown. The modulator consists of a circulator and a varactor diode mount. The reflection coefficient has been measured at $9.45 \mathrm{GHz}$, the specified frequency of the modulator, and the result is shown in Fig. 5 with the varactor bias voltage as a parameter. Since the binary $180^{\circ}$ modulation gives the lowest modulation loss, the modulator is driven so that it takes "on" and "off" states in every half-period of the modulation frequency, which is $1.8 \mathrm{MHz}$. The Fourier coefficients estimated from the reflection coefficient waveforms are

$$
a_{1}=0.955 \quad b_{1}=0.143 \quad \theta_{1}-\phi_{1}=182.46^{\circ}
$$

where the departure of $\theta_{1}-\phi_{1}$ from $\pi$ is due to the transient behavior of the reflection coefficient during the transitional period between the binary states.

Three circulators inserted between the modulator and the mixer prevent the homodyne carrier from leaking into the signal channel [2]. The total inverse isolation between them is about $140 \mathrm{~dB}$, while the total insertion loss of the circulators is $1.1 \mathrm{~dB}$. The mixer employs two low-noise GaAs Schottky diodes arranged in a balanced configuration. The conversion loss of the mixer is $4.7 \mathrm{~dB}$. The noise figure $F_{\text {ree }}$ for the combination of the mixer and the IF amplifier is $7 \mathrm{~dB}$.

The conversion loss of the system is measured by the direct method; the microwave signal power is measured 


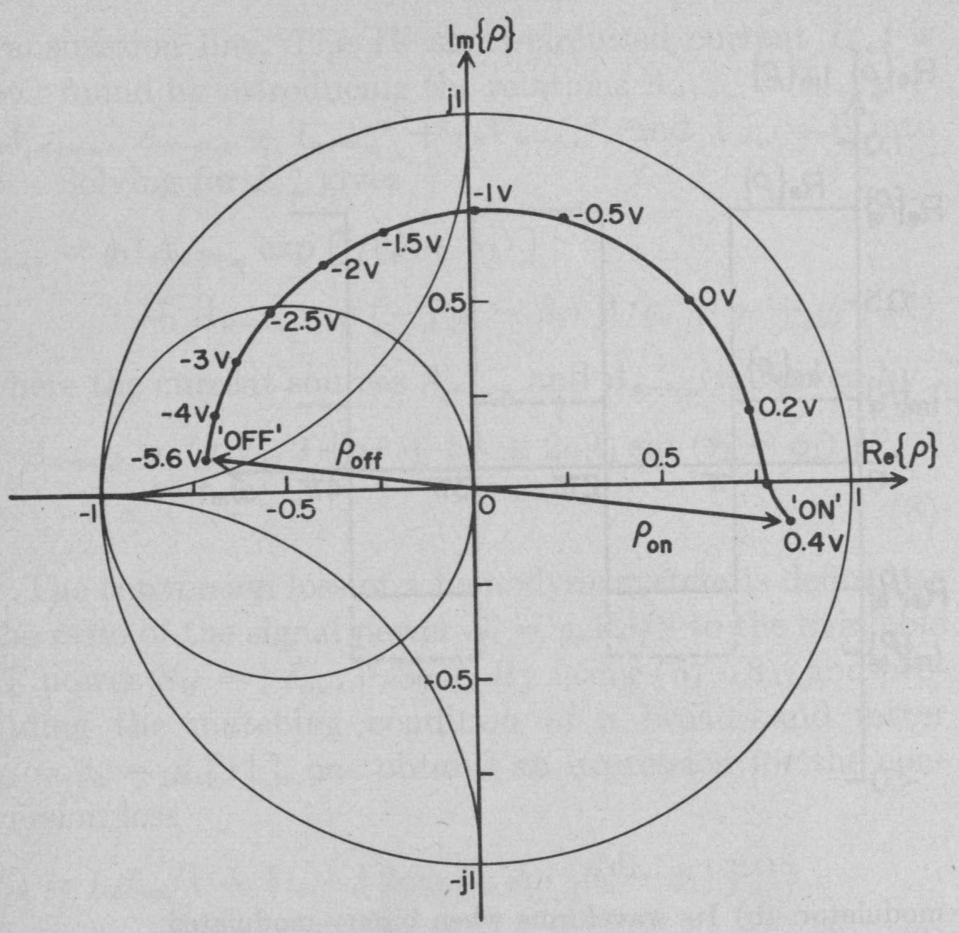

Fig. 5. The reflection-coefficient locus of the varactor modulator measured at $9.45 \mathrm{GHz}$.

by a bolometer, and the IF available power is measured by a spectrum analyzer. The results are plotted in Fig. 6 against the phase difference between the homodyne carrier and the signal, which is introduced by means of a variable phase shifter in the carrier channel. The phase difference on the abscissa is a relative value to the reading of the phase shifter when the maximum conversion loss is obtained, which is conveniently taken as $\pi / 2$. The solid curve shows the conversion loss calculated by (9), using the Fourier coefficients given in (18) and taking the insertion loss of the circulators into account.

At minimum conversion loss the noise figure of the system is estimated by measuring the signal power required to double the zero-signal detected output. The measured value at $9.45 \mathrm{GHz}$ is $8.7 \mathrm{~dB}$, while that calculated by (16), including the insertion loss of circulators, is $8.14 \mathrm{~dB}$.

By way of comparison, the same modulator is driven by the sinusoidal source so that it continuously traces out the reflection coefficient locus shown in Fig. 5. The measured values of the minimum conversion loss and the quadrature signal rejection ratio are $9.8 \mathrm{~dB}$ and $34 \mathrm{~dB}$ at $9.45 \mathrm{GHz}$, while the values calculated by (9), using the estimated Fourier coefficients, are $9.6 \mathrm{~dB}$ and $36 \mathrm{~dB}$, respectively. Comparison of these results with corresponding values of $6.3 \mathrm{~dB}$ and $42 \mathrm{~dB}$ shown in Fig. 6 shows the superiority of the binary $180^{\circ}$ modulation in obtaining both high phase- and amplitude-detection sensitivities, as described in the previous section.

\section{CONCLUSIONS}

The phase-sensitive conversion loss and noise figure of the homodyne system employing a signal modulation technique have been theoretically derived. Investigation

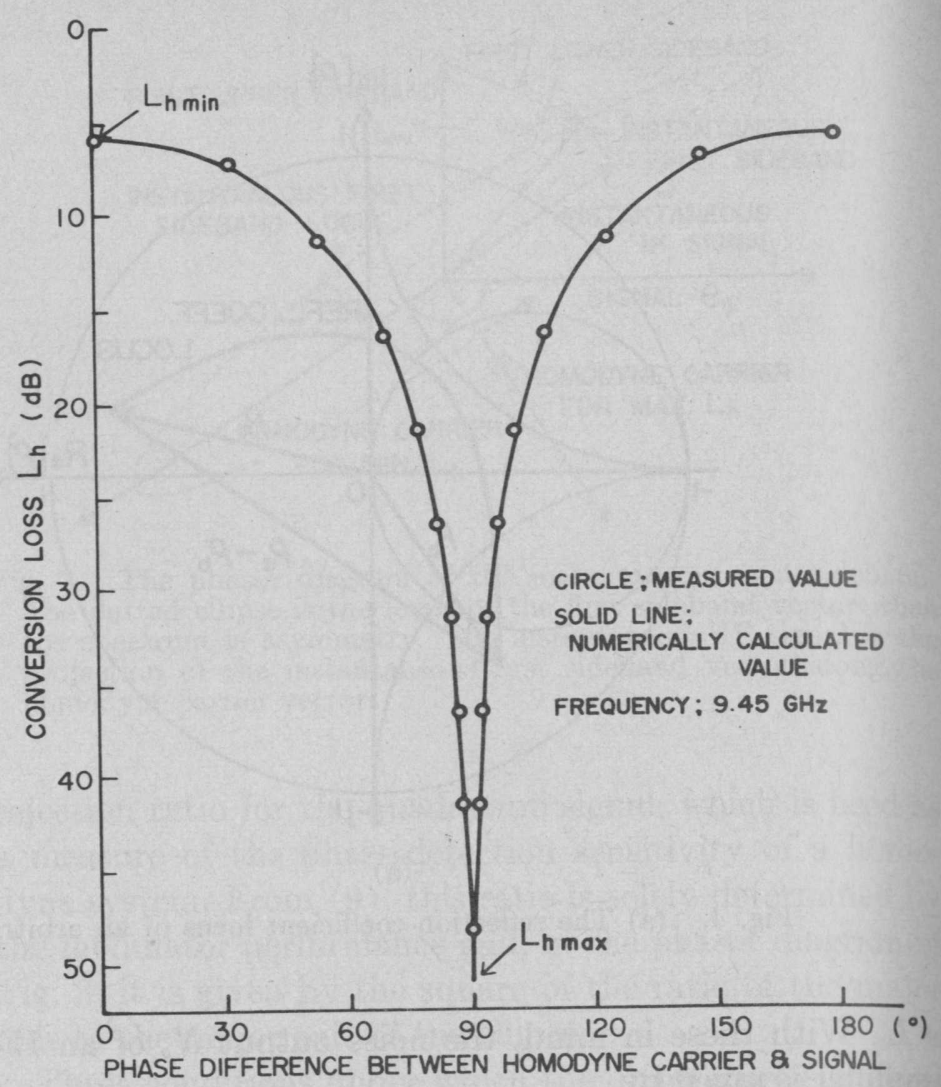

Fig. 6. The conversion loss plotted against the phase difference between the signal and the homodyne carrier.

of the modulation method for the homodyne system using resultant expressions shows that the binary modulation method is optimum in obtaining both the highest phaseand highest amplitude-detection sensitivities. The theoretical results have been confirmed experimentally by the practical homodyne system employing a varactor binary modulator. This system has a conversion loss and a noise figure comparable with those of a conventional heterodyne system; it is widely applicable to all sorts of microwave spectrometers. A further reduction of the conversion loss is possible using a high-quality and high-speed p-i-n diode recently available as a modulating device.

\section{APPENDIX}

\section{MODULATION LOSSES FOR AMPLITUDE, PHASE, AND BINARY MODULATIONS}

\section{A. Amplitude Modulation}

The reflection-coefficient locus of an amplitude modulator is shown in Fig. 7(a). The reflection coefficient $\rho$ is given by

$$
\rho=\frac{1}{2}\left(\rho_{a}+\rho_{b}\right)+\frac{1}{2}\left(\rho_{a}-\rho_{b}\right) \cos \omega_{m} t .
$$

Thus the modulation loss is given by

$$
\left[L_{m}\right]_{\mathrm{AM}}=\left(2 \sqrt{2} / \rho_{a}-\rho_{b}\right)^{2}
$$

while the modulation loss when binary modulated is given by 


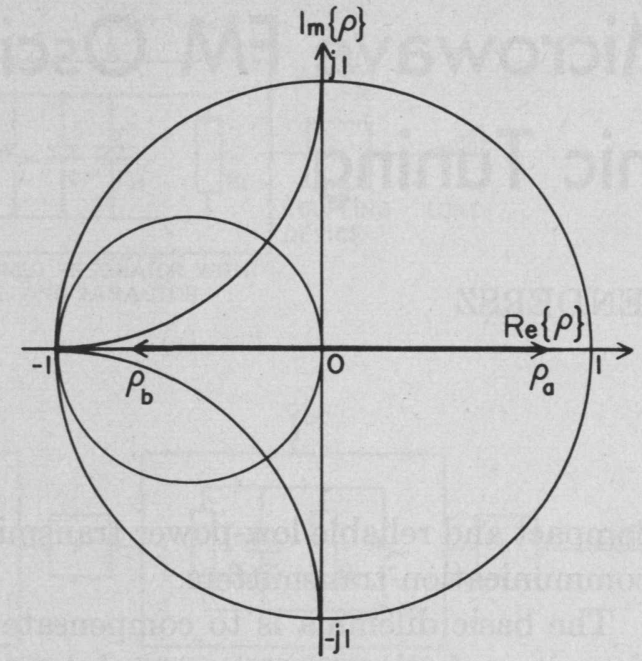

(a)

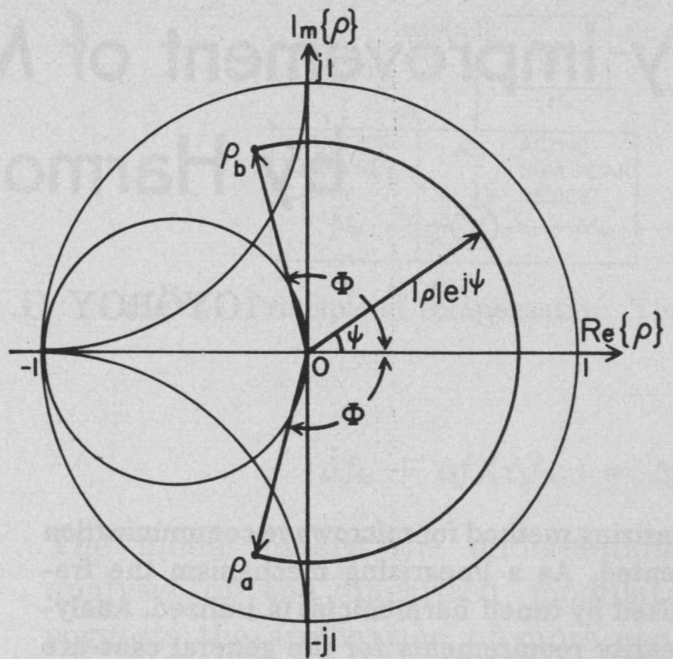

(b)

Fig. 7. The reflection coefficient loci of (a) amplitude and (b) phase modulators.

$$
\left[L_{m}\right]_{\mathrm{BM}}=\left[\pi / \sqrt{2}\left(\rho_{a}-\rho_{b}\right)\right]^{2} .
$$

From (A-2) and (A-3), it is evident that $\left[L_{m}\right]_{\mathrm{BM}}$ is always smaller than $\left[L_{m}\right]_{\mathrm{AM}}$.

\section{B. Phase Modulation}

The reflection-coefficient locus of a phase modulator is shown in Fig. 7 (b). The reflection coefficient $\rho$ is given by $\rho=|\rho| \exp \left(j \Phi \cos \omega_{m} t\right)$

$$
\begin{aligned}
& =|\rho|\left\{J_{0}(\Phi)+2 \sum_{n=1}^{\infty} J_{2 n}(\Phi) \cos \left(2 n \omega_{m} t+n \pi\right)\right. \\
& \left.+j 2 \sum_{n=1}^{\infty} J_{2 n-1}(\Phi) \cos \left[(2 n-1) \omega_{m} t+(n-1) \pi\right]\right\}
\end{aligned}
$$

where $J_{n}(\Phi)$ is the Bessel function of the first kind, of order $n$ and argument $\Phi$, and $\Phi$ is the maximum phase deviation. Thus the modulation loss is given by

$$
\left[L_{m}\right]_{\mathrm{PM}}=1 /\left[\sqrt{2}|\rho| J_{1}(\Phi)\right]^{2}
$$

while the modulation loss when binary-modulated is given by

$$
\left[L_{m}\right]_{\mathrm{BM}}=(\pi / 2 \sqrt{2}|\rho| \sin \Phi)^{2}
$$

where $\Phi$ is always smaller than $1.85 \mathrm{rad}$ at which $J_{1}(\Phi)$ becomes maximum. In this range of $\Phi,(\mathrm{A}-6)$ is smaller than (A-5).

\section{ACKNOWLEDGMENT}

The authors wish to thank M. Ashiki for his help in the construction of the system and the measurements.

\section{REFERENCES}

[1] G. E. Schafer and R. R. Bowman, "A modulated sub-carrier technique of measuring microwave attenuation," Proc. Inst. Elec. Eng., vol. 109, pt. B, suppl. 23, pp. 783-786, May 1962.

[2] B. B. O'Brien, "A pseudo-superheterodyne receiver for measuring phase and amplitude," IEEE Trans. Instrum. Meas., vol. IM-16, pp. 124-128, June 1967.

[3] S. B. Cohn and N. P. Weinhous, "An automatic microwave phase-measurement system," Microwave J., vol. 7, pp. 49-56, Feb. 1964

[4] T. Hayashi and I. Takao, "Application of the switching-homodyne reception technique to the ESR spectrometer" (in Japanese), Bull. Res. Inst. Electron., Shizuoka Univ., Hamamatsu, Japan, vol. 2, pp. 37-46, June 1967.

[5] E. A. Faulkner and P. W. Whippey, "Electron-spin-resonance spectrometer using high frequency amplitude modulation," Proc. Inst. Elec. Eng., vol. 113, pp. 1159-1162, July 1966.

[6] W. E. Little, "Further analysis of the modulated subcarrier technique of attenuation measurement," IEEE Trans. Instrum. Meas., vol. IM-13, pp. 71-76, June-Sept. 1964.

[7] R. J. King and R. I. Christopherson, "A homodyne system for the measurement of microwave reflection coefficients," $I E E E$ Trans. Microwave Theory Tech. (Corresp.), vol. MTT-18, pp. 658-660, Sept. 1970.

[8] B. A. Howarth and T. J. F. Pavlásek, "Analysis of automatic homodyne method amplitude and phase measurements," IEEE Trans. Microwave Theory Tech. (Short Papers), vol. MTT-20, pp. 623-626, Sept. 1972.

[9] S. Stuchly, A. Kraszewski, and M. Rzepecka, "Microwaves for continuous control of the industrial process," Microwave J., vol. 12, pp. 51-57, Aug. 1969.

[10] J. Kaliński, "Further possibilities of the modulated subcarrier technique for microwave attenuation measurements in industrial applications," IEEE Trans. Instrum. Meas. (Short Papers), vol. IM-21, pp. 291-293, Aug. 1972.

[11] H. C. Torrey and C. A. Whitmer, Crystal Rectifiers. New York: MeGraw-Hill, 1948, pp. 111-157.

[12] P. F. Panter, Modulation, Noise, and Spectral Analysis. New York: McGraw-Hill, 1965, pp. 206-212. 